\title{
humanidades
}

Revista humanidades

Enero-junio, 2016 • Volumen 6, número 1 • ISSN 2215-3934・pp. 1-48

\section{La Universidad en España y Colombia en la era de la globalización}

DOI: http://dx.doi.org/10.15517/h.v6i1.25116

\section{Santiago Ospina García}

Licenciado en Enseñanza de Lenguas Extranjeras por la Universidad de Antioquia (Colombia). Docente y lingüista, estudiante de doctorado en Estudios Hispánicos en la Universidad Paris Ouest Nanterre La Défense (Francia). Correo electrónico: santiago.ospinagarcia@sciencespo.fr

\section{Mercè Pujol Berché}

Profesora Catedrática de Español y Catalán (Lengua y Civilización) en la Universidad París Ouest Nanterre La Défense (Francia). Codirectora del CRIIA (Centro de investigaciones ibéricas e iberoamericanas) de la misma universidad. Correo electrónico: mercepujolberche@gmail.com 


\title{
La Universidad en España y Colombia en la era de la globalización
}

\section{Resumen}

En el presente artículo se revisan los asuntos más relevantes relacionados con la Universidad en España y Colombia actualmente. A pesar de sus diferencias, ambos países comparten problemáticas comunes en el mundo globalizado, de economía de mercado y de "economía del conocimiento". Las consecuencias de las políticas económicas neoliberales creadas por organizaciones supranacionales como la OCDE y la Comisión Europea, aplicadas en estos países, resultan nefastas para sus servicios públicos y sistemas educativos. En este contexto, se proponen algunas reflexiones críticas sobre el estado actual de la Universidad en ambos países, las causas que han llevado al debilitamiento de esta y el papel que juegan las políticas de Estado en la reproducción de las desigualdades sociales y en la pauperización de la sociedad.
Palabras clave:

Globalización, universidad, políticas económicas, organizaciones supranacionales, desigualdades sociales.

\section{Spanish and Colombian Universities in the Age of Globalization}

\begin{abstract}
This article deals with the most relevant events concerning Higher Education in Spain and Colombia nowadays. Despite their differences, both countries share common issues in a globalized world controlled by the market and the knowledge economy. The consequences of neoliberal economic policies created by supranational organizations such as the OECD and the European Commission and implemented in these countries have been disastrous for their public services and educational systems. In this context, we propose a number of critical observations about the current state of Higher Education in both countries, the causes that have contributed to weakening their higher education systems and the role played by the public policies in reproducing social inequalities and triggering the pauperization of the society.
\end{abstract}

Keywords: Globalization, higher education, economic policies, supranational organizations, social inequalities. 
Peters (2005) asegura, partiendo de los trabajos de Bill Readings (1996) y de Jean François Lyotard (1984, 1992, 1995), que la universidad de la era moderna está fundada en tres ideas: la razón (de Kant), la cultura (de Humboldt) y la excelencia (de la tecno burocracia). Empero, los discursos que fundaron la universidad moderna, la razón y la cultura, han sido quebrantados por el nuevo discurso sobre la "excelencia", que refleja la lógica del rendimiento impuesta por la globalización y las ciencias de la gestión y del mercadeo. Los nuevos valores impuestos amenazan permanentemente con cambiar la misión de la universidad y hacer desaparecer tanto las libertades académicas como la autonomía, las cuales han sido los pilares tradicionales de las universidades liberales; así, las Universidades están en proceso de transformación profunda desde hace décadas.

El modelo napoleónico encomendaba a la universidad la tarea de la transmisión de conocimientos científicos; por lo tanto, el foco estaba en la docencia. En el modelo de Humboldt, el principal quehacer de la universidad era la investigación (la cultura). Actualmente se ha adoptado el modelo llamado de Newman, en el que la vocación de la universidad es formativa. Se puso en marcha en Irlanda, a finales del siglo XIX; esta visión de la universidad se está imponiendo de forma considerada, puesto que la línea directriz hoy es el aprendizaje a lo largo de toda la vida. Los estudiantes pueden aprender en clase (magistral o de otro tipo), pero sobre todo tienen que ser responsables de su propio aprendizaje, el cual realizan de diferentes formas (clases, seminarios, prácticas, trabajo en grupos, exposiciones orales, etc.), ya que el objetivo que se le da a la universidad es que los egresados sean capaces de enfrentarse a todos los retos que puedan surgir en la sociedad de hoy.

\section{Introducción}


La universidad española ha sufrido cambios importantes desde los años sesenta del siglo pasado, pero sobre todo a partir del último cuarto de siglo. Esta se ha democratizado, es decir que accede a esta un número más importante de estudiantes que en épocas anteriores; para algunos, esta democratización es sinónimo de masificación. Pero a pesar de ello sigue estando fuertemente estratificada al menos geográficamente, ya que las comunidades autónomas de Madrid y de Cataluña tienen la cuarta parte de los centros, el $40 \%$ del profesorado y estas se dirige el $40 \%$ del alumnado. Es cierto también que las mujeres han accedido a los estudios superiores; sin embargo, a pesar de esta feminización (en algunas carreras más que en otras $^{1}$ ), la estratificación por género sigue vigente, ya que en las carreras de la salud ellas representan el 70,1 \%, mientras que en las de ingeniería y de arquitectura representan el 26,10 \% (cf. Ministerio de Educación, Cultura y Deporte, 2013). También sigue sin haber paridad en el cuerpo docente, ya que solo el $13 \%$ ocupa una cátedra, mientras que casi la tercera parte de los hombres ocupa estos puestos $(27,7 \%)$.

Los cambios empezaron con la reforma universitaria de 1983, siguieron en los años noventa con la creación de nuevas universidades - muchas de estas privadas-, continuaron en 1999 con la firma del plan Bolonia y en el 2007 con la reforma de financiación. Más tarde, con el estallido de la crisis (que aún no se ha superado) y la globalización, continuaron las transformaciones, pero esta vez el conjunto del país se ha visto afectado. La globalización trajo consigo cambios estructurales de envergadura cuyas consecuencias empiezan a sentirse en las nuevas misiones que se le van asignando a la universidad, en la gobernanza empresarial y en la monedarización del conocimiento. 
De este modo, la educación y el conocimiento se han convertido en valores de intercambio monetario y de comercialización; "todo se compra y se vende". Dicho de otra forma, se persigue que las universidades funcionen como empresas y que el aumento de los universitarios suponga un aumento de los ingresos para las instituciones y, por lo tanto, una disminución del dinero público transferido a las mismas. La Universidad fomenta actualmente la cultura emprendedora, aumenta su relación con las empresas y otorga mayor importancia a las capacidades, las llamadas competencias exigidas por el mundo laboral, aunque la inserción laboral no depende solo de las competencias adquiridas en la universidad o en las prácticas que algunas formaciones exigen, sino en la salud del mercado laboral, la cual depende de las políticas económicas que se implementen a nivel global y local. Así pues, las competencias deben, además, acompañarse de conocimientos y de saberes en las que deben asentarse; no hay que olvidar que una de las acepciones de la palabra competencia es la rivalidad entre empresas que luchan por situarse en primera posición o por vender un producto.

En lo referente a Colombia, estos factores son igualmente relevantes, aunque el proceso de decadencia empezó antes que en España. En efecto, en este país la Universidad (y todo el servicio público en general) viene deteriorándose desde los años sesenta como consecuencia de la aplicación de políticas neoliberales impuestas por organizaciones internacionales con la complicidad de los gobiernos de turno. Estas políticas no han hecho más que agudizar la miseria y la inseguridad en el país. Así, a pesar de que en los últimos doce años Colombia ha logrado un crecimiento económico muy importante, esa prosperidad no ha servido para reducir las injusticias sociales; al contrario, hoy la brecha entre los ricos y los pobres parece más 
grande y gran parte de la población debe autofinanciar su salud y su educación. La proliferación de instituciones de educación superior privadas y de pésima calidad es precisamente una de las consecuencias del desfinanciamiento de la educación pública por parte del Estado. En tal contexto, no queda duda de que las instituciones más serias son las tradicionales, es decir las universidades públicas y algunas privadas. No obstante, en este país suramericano las universidades privadas representan no solo una opción, sino también una solución cuando no se puede estudiar en un plantel público, siempre y cuando se tenga los medios económicos para acceder a ellas.

Para mayor claridad, expondremos de forma separada, lo más relevante en España y en Colombia teniendo en cuenta que las diferencias entre ambos países impiden que se traten los temas exactamente de la misma forma. Por ende, hemos dividido el artículo en cuatro partes que exponen otros tantos aspectos que consideramos los más relevantes, atendidas las situaciones particulares de ambos países en cuanto al propio sistema universitario y las consecuencias sufridas por cuenta de la globalización y del impacto del neoliberalismo en la sociedad del conocimiento. En la primera parte se expone un panorama general del sistema de educación en ambos países, poniendo el énfasis, para España, en los cambios que cada gobierno ha provocado en el sistema universitario y en la investigación científica; en lo referente a Colombia, el acento se pone en el aumento de las desigualdades sociales en el seno de un sistema que lleva años en crisis. En la segunda parte observamos cómo el compromiso de los Estados y, por lo tanto, de lo público, está desapareciendo poco a poco, pero de forma constante en un ámbito tan crucial para la Humanidad como es el de la educación y el de la formación; esta desatención que incrementa las desigualdades y 
dificulta el acceso de las clases más desfavorecidas al alma mater. La tercera parte aborda la relación entre el valor de las matrículas y el rendimiento de los estudiantes, que no es siempre una relación de causaefecto, sino de correlación. Se menciona también la excelencia, en el sentido de calidad y de singularidad para distinguirla de la excelencia vendida como ranking competitivo. En la cuarta y última parte se aborda el tema de la aparición de la llamada "sociedad del conocimiento" provocada por la globalización, el mercadeo de todo lo relacionado con él y los falsos argumentos usados sobre la empleabilidad de los estudiantes, el mundo laboral y la situación de paro y de crisis que se vive en la actualidad.

El nombre tan solo del Ministerio del cual depende la universidad y con ella la educación superior y la investigación que se realiza en la misma nos da indicios de la importancia que un país otorga a estos temas que, en nuestra opinión, son cruciales para los individuos. Desde la muerte de Franco en 1975 hasta la actualidad, el Ministerio de tutela ha cambiado 12 veces de nombre. La designación no es ni pura denominación, ni anodina, al contrario, ya que según el nombre que se le da, podemos apreciar las tareas que le son encomendadas y la importancia que se le otorga a la ciencia, a la educación, al deporte, a la cultura o incluso a la innovación. Al sustantivo educación que aparece casi siempre en el nombre del Ministerio, se la ha ido añadiendo, la mayor parte de las veces, el sustantivo

\section{Panorama del sistema de educación en España y Colombia}

\section{España: El baile de nombres del Ministerio y de sus titulares} ciencia, aunque a veces ha sido reemplazado por el de cultura y otras veces por el de deporte. De este modo, el Ministerio que empezó llamándose en 1975 de Educación y Ciencia, actualmente toma los otros dos sustantivos mencionados, cultura y deporte, mientras que la ciencia se ha ido hacia otro ministerio, el de Economía y Competitividad, con una Secretaría de Estado 
(de menor rango que Ministerio) denominada de Investigación, Desarrollo e Innovación.

El controvertido ministro de Educación, Cultura y Deporte, José Ignacio Wert nombrado por Mariano Rajoy cuando el Partido Popular llegó al poder en 2011, ha sido sustituido por Íñigo Méndez de Vigo hace apenas unas semanas sin demasiados miramientos por quien le nombró. Luís de Guindos sigue con su cartera en el Ministerio de Economía y Competitividad, mientras que Carmen Vela (empresaria e investigadora (no doctora) en bioquímica) está al frente de la Secretaría de Estado de Investigación, Desarrollo e Innovación. A ella se le debe esa frase algo fuera de lugar teniendo en cuenta el cargo que ocupa, publicada en un artículo de Nature escrito por ella misma titulado “Turn Spain's Budget crisis into an opportunity" en el que sostenía que "Tenemos que reducir la cantidad de investigadores para mejorar la calidad de los contratos. Tenemos que hacer esto de todos los modos: el sistema español de I+D no es suficientemente grande para justificar el gasto en los actuales investigadores"2. Lo más importante para ella es el "gasto" y la "reducción del número de investigadores", no el mantenerlos (son evaluados regularmente por la ANECA -Agencia Nacional de Evaluación de la Calidad y Acreditación- y otros organismos) en mejores condiciones (contratos más largos o incluso convirtiéndolos en funcionarios).

Después del largo periodo franquista, la universidad española necesitaba, sin duda alguna, cambios profundos y estructurales. Con la llegada de Felipe González al poder, en 1982, el Ministerio cuyo titular era en ese momento Federico Mayor Zaragoza, vuelve a su nombre de origen, Ministerio de Educación y Ciencia. Son nombrados en los diferentes mandatos personas de su gran confianza como Javier Solana o Alfredo 
Pérez Rubalcaba y se promueve una serie de leyes estructurales de importancia. España entra en 1983 en el CERN (Organización Europea de Investigación nuclear) ubicada en la frontera francosuiza, cerca de Ginebra, lo que significa un paso importante para poner a la ciencia española en las esferas europeas. José María Maravall realiza un paso de gigante al promover ese mismo año, la Ley de Reforma Universitaria (25.08.83), llamada “ley Maravall”, en la que se otorga autonomía a las universidades, se reestructura la organización docente (se pone sobre la mesa el tema de la endogamia, aspecto recurrente en la universidad española) y se producen cambios profundos en los planes de estudio. Ese mismo año tiene lugar el traspaso de competencias. En 1986, se promueve la Ley de la Ciencia (18.04.1986) para fomentar e impulsar la investigación científica a través de la Coordinación General de la Investigación Científica y Técnica. Otro paso importante no exactamente para la ciencia en especial, aunque sí para la construcción europea, es la puesta en marcha del Programa Erasmus (15.05.1987), que acaba de cumplir 28 años.

Los años noventa, en época de plena bonanza económica, vieron la creación de Universidades, algunas realmente de nueva creación, como la Universidad Pompeu Fabra en Barcelona o la Universidad Carlos III en Madrid, que consiguieron grandes cantidades de dinero en sus inicios y que contaron con personal docente y científico de renombre. Otras nacieron de una separación, fue el caso, por ejemplo, de la Universidad Rovira i Virgili de Tarragona que dependió de la Universidad de Barcelona, como Campus de Tarragona hasta el año 1992. Durante esos mismos años, las Universidades privadas aparecieron con fuerza, algunas, de la Iglesia católica como la Universidad Ramon Llull en Cataluña, que se constituyó en 1990 a partir de centros ya existentes como la Escuela de Magisterio 
Blanquerna fundada en 1948 y adscrita posteriormente en 1979 a la Universidad de Barcelona; otras vinculadas al Opus Dei, como la Universidad de Navarra que cuenta con centros en Pamplona, San Sebastián, Madrid y Barcelona.

Es de obligada mención la creación de universidades privadas ya que representan el tercio de las universidades españolas. Mención especial merece la primera aprobada por las Cortes generales, la Universidad Alfonso X el Sabio en la Comunidad de Madrid, fundada en 1993, que se autodenomina "la Universidad de la Empresa" porque explícitamente es un "proyecto empresarial" cuya finalidad es que sus egresados ingresen rápidamente en el mundo laboral. Esto último ilustra lo que queremos desarrollar en nuestra contribución, a saber, cómo la Universidad, con sus transformaciones y reformas sin cesar, se está convirtiendo en una empresa dejando de lado su función inicial que es la de pensar, formar, reflexionar, instruir y desarrollar un espíritu crítico; sin olvidar, como no, la función de investigar, es decir la de realizar actividades intelectuales y experimentales de forma sistemática para aumentar el conocimiento en una disciplina dada. Cuando las Universidades se convierten en empresas a través de su gobernanza y del espíritu competitivo que se crea en ellas para dar más valor al "stock de capital humano" (como así lo denomina el propio Ministerio (ídem, p. 134), su función cambia y se convierten en organizaciones de prestación de servicios con fines lucrativos. Se busca para los estudiantes, la utilidad de la formación recibida en la Universidad para acceder al mercado de trabajo y para el cuerpo docente, se establece una relación (a veces malsana) entre la productividad científica (los resultados científicos) y las recompensas académicas, como mencionaremos unas líneas más abajo. Otra reforma sobre la financiación tiene lugar en 1995. 
La llegada al poder del Partido Popular (PP) con José María Aznar como Presidente trae algunos cambios, como el nombre mismo del Ministerio, ya que a la educación se le añade cultura, Ministerio de Educación y Cultura. Se nombra como titulares a "pesos pesados" del PP como la que ha sido durante muchos años Presidenta de la Comunidad de Madrid,

Esperanza Aguirre, o al que es actualmente Presidente español, Mariano Rajoy. Los ministros de educación firman, en $1999^{3}$, el llamado plan Bolonia para la puesta en marcha del Espacio Europeo de Educación Superior-EEES- con la finalidad de generar un espacio único y armonizar los diferentes sistemas educativos para que haya movilidad entre los estudiantes y puedan realizar sus estudios en cualquier país (de los 47 que componen actualmente el EEES), es decir dotar de transparencia en términos de legibilidad a las universidades (y posteriormente a los empleadores). Otro objetivo es la intensificación de los procedimientos de calidad. Otra de las finalidades del plan Bolonia es el de erradicar que la Universidad viva al margen de las necesidades del mercado de trabajo, se quiere facilitar la empleabilidad de los estudiantes, es decir dotarla de carácter profesionalizador, en otras palabras, la Universidad debe proveer al estudiante un futuro profesional más amplio. No se busca, según fuentes del propio Ministerio, "la adecuación para un oficio concreto sino la adquisición de conocimientos junto con la formación en destrezas como la capacidad de análisis y de síntesis, de resolver problemas o de aplicar conocimientos adquirido a la práctica en su campo de estudio" (cf. Ministerio de Educación, Cultura y Deporte, 2013, p. 65).

El llamado informe Bricall sobre universidades se publica en el año 2000. Es sobre todo durante el segundo mandato de José Ma Aznar con mayoría 
absoluta, que los cambios afectan más la ciencia y se "ficha", en el año 2000, a personalidades que vienen de la empresa como Anna Virulés. Se pretende incrementar, gracias a los buenos resultados económicos que ha obtenido Aznar, la presencia de la empresa (y de sus fondos) en la universidad y seguir situando "España en el mundo como primera potencia" (los grandes sueños de Aznar), aunque España sigue siendo una potencia media. Promueve una ley importante como la Ley Orgánica 6/2001 (21.12.2001) y se crea la Fundación española para la Ciencia y la Tecnología (FECYT, 27.04.2001).

Con la llegada de José Luis Rodríguez Zapatero, el Ministerio vuelve a ser de Educación y Ciencia. Promueve la Ley Orgánica 4/2007 (12.04.2007) que modifica la Ley Orgánica 6/2001 (21.12.2001) y llega otra nueva reforma de financiación en el mismo año 2007. En su segundo mandato, en 2008, va a crear el Ministerio de Ciencia e Innovación que bajo las órdenes de Cristina Garmendia pretende dar un gran impulso a la investigación y a través de ella a la innovación. El informe solicitado a la $\mathrm{OCDE}^{4}$ se publica en 2008. Cuando la crisis empieza a puntear, aunque la sigue negando Zapatero, éste crea el Ministerio de Educación, Política social y Deporte que tiene una vida muy corta (menos de 2 años), para volver, en 2009, al punto de partida, solicitando al catedrático de filosofía y rector de la Universidad Autónoma de Madrid, Ángel Gabilondo para que asuma su titularidad. Una nueva reforma de financiación se lleva a cabo en 2010, mientras una serie de expertos internacionales publican el mismo año, "Audacia para llegar más lejos". En 2011, se promueve la ley de la Ciencia, la Tecnología y la Innovación: I+D+i que sustituye a la ley de 1986. 
La llegada de Mariano Rajoy en 2011 conlleva las medidas anunciadas por él el 19 de diciembre de 2011 y confirmadas en su discurso de investidura (30 de diciembre). Lo principal es la austeridad presupuestaria (austeridad y eficacia son las dos palabras clave en la administración) con medidas urgentes en economía y presupuestos (los recortes que ya habían empezado en la época de Zapatero). La restructuración general concierne sobre todo a la sanidad y a la educación. La competitividad económica está a la orden del día. Rajoy anunció la creación de una Comisión de Expertos para impulsar una profunda reforma del sistema universitario. Reforma que quiere alcanzar tres objetivos: mejorar el rendimiento de los alumnos (según el Gobierno solo el 80 \% de los matriculados acaba la carrera), fijar especializaciones y lograr una mayor flexibilización de las ofertas para evitar duplicidades. El Presidente y su ministro José Ignacio Wert se preguntaron por qué en España, las "tantas" universidades públicas no ocupan los primeros puestos de los rankings internacionales ${ }^{5}$. Una de sus cuestiones recurrentes es ver lo que sobra: campus, alumnos, títulos, profesores, etc. No se preguntan desafortunadamente lo que le falta a la universidad para ofrecer un servicio de calidad tanto en enseñanza como en investigación.

No es por lo tanto en absoluto anodino, como decíamos al inicio, que desde 2011 hasta la actualidad, la faceta de investigación e innovación dependa del Ministerio de Economía (el que atribuye todos los presupuestos a todos los ministerios). Tampoco es anodino que dicho Ministerio se denomine por primera vez de Competitividad y que, en algunas declaraciones, la Secretaria de Estado, Carmen Vela, sostenga que hay demasiados investigadores, que resultan muy caros. Algo fundamental para un país como son esos tres factores -investigación, desarrollo e innovación-, se 
ponen conjuntamente con la competición. Dicho de otra forma, esto conjuga con el proyecto neoliberal del cual la Universidad, tal y como lo recuerda Marsi (2010), es indispensable para las élites. Las universidades (y el cuerpo docente) compiten entre ellas (siguiendo el modelo americano), se convierten en rivales para conseguir un mismo empeño y esos fondos tan preciados. En un contexto de globalización, de neoliberalismo ${ }^{6}$ y de monedarización de la educación y del conocimiento, la crisis económica que azota España desde 2007 no ha hecho si no fortalecer el sistema actual, agrandar el abismo entre las clases sociales y pauperizar a la población. A esta crisis económica, hay que añadir la crisis del Estado-nación y la crisis de la democracia ya que la gente no se ve representada por la clase política, salpicada de corrupción, incluso institucional. Rajoy privatiza y la gente sale a la calle y vota $^{8}$.

Muy al estilo de lo que sucede en Europa, en términos generales los ministros colombianos están obligados a seguir las órdenes impuestas por organismos supranacionales (FMI, BM, OCDE, etc.) que no son elegidos por los pueblos y que no le tienen que rendir cuentas a nadie. Así pues, desde los años sesenta Colombia viene acatando las órdenes de estas organizaciones (Cruz Atehortúa, 2012), lo cual deja poco margen de maniobra a los funcionarios que deseen llevar a cabo políticas contrarias a las que se les "recomiendan".

En 2013, la misión formulada por el Ministerio era la de "Garantizar el derecho a la educación con criterios de equidad, calidad y efectividad, que forme ciudadanos honestos, competentes, responsables y capaces de construir una sociedad feliz, equitativa, productiva, competitiva, solidaria y orgullosa de sí misma”. En $2015^{9}$ se puede leer $^{10}$ que el objetivo es "lograr una EDUCACIÓN DE CALIDAD, que forme mejores seres

\section{Colombia: Un sistema en crisis que reproduce las desigualdades sociales}


humanos, ciudadanos con valores éticos, competentes, respetuosos de lo público, que ejercen los derechos humanos, cumplen con sus deberes y conviven en paz. Una educación que genere oportunidades legítimas de progreso y prosperidad para ellos y para el país. Lograr una educación competitiva, pertinente, que contribuya a cerrar brechas de inequidad y en la que participa toda la sociedad". En cuanto a su misión, el Ministerio afirma que: “en 2014 Colombia será uno de los países con la mejor calidad de educación en América Latina, habrá reducido significativamente las brechas de inequidad y será modelo de eficiencia y transparencia a nivel nacional e internacional". Sin embargo, como sucede con la mayoría de los asuntos bajo la tutela del Estado colombiano, existe una brecha descomunal entre la teoría y la práctica, entre lo que dicen hacer o querer hacer los funcionarios en las oficinas y lo que pasa realmente en las ciudades y en el campo. Basta con mirar los documentos e informes de cualquier ONG seria o de la ONU para darse cuenta de que los buenos deseos del gobierno generalmente son letra muerta. Así, a pesar del crecimiento económico y de los golpes asestados a las diferentes guerrillas (para muchos ciudadanos estas son las causantes de las desgracias del país), Colombia no ha dejado de producir corrupción, miseria, inseguridad y muertos en todo el territorio nacional.

A manera de ejemplo, en el ámbito de la educación, pocos de los objetivos antes mencionados han sido conseguidos ya que los resultados de Colombia en los rankings internacionales están dentro de los peores de América Latina y del mundo. En efecto, todos los informes de organizaciones internacionales, documentos académicos y artículos periodísticos que hemos revisado en aras de la redacción de este texto, sin que importe la ideología política o económica de los autores, señalan que 
el sistema de educación colombiano está en crisis desde hace décadas por diversas razones. Los únicos informes que intentan maquillar o minimizar estas evidencias son los publicados por el gobierno y sus diversas instituciones, por las organizaciones internacionales (OCDE, Banco Mundial, Fondo Monetario Internacional, Banco Interamericano de Desarrollo, etc.) o por algunos periódicos (The Wall Street Journal, The Financial Times) y periodistas (Oppenheimer, 2010) liberales.

No obstante, la realidad del día a día de la educación en Colombia deja sin fundamento cualquier intento de manipulación de la verdad. De hecho, para comenzar, se pueden evocar las desalentadoras cifras proporcionadas por la misma OCDE. El Programa para la Evaluación Internacional de Alumnos de la OCDE (PISA, por sus siglas en inglés: Programme for International Student Assessment) es una encuesta del conocimiento y las habilidades que se realiza cada tres años a estudiantes de 15 años. Se ha diseñado para poder hacer comparaciones válidas entre países y culturas. PISA 2009 y 2012 se centra en particular en la lectura, pero también cubre las matemáticas y las ciencias. Asimismo, hay una prueba de solución creativa de problemas (prueba complementaria). La escala de desempeño de PISA está construida de forma que para cada una de las tres áreas, la puntuación media de los países OCDE se sitúa en torno a 500, con aproximadamente dos tercios de estudiantes que puntúan entre 400 y 600 puntos. Una diferencia de 39 puntos equivale a un año de escolaridad. En el área principal de la prueba de 2009, la lectura, los estudiantes colombianos lograron una puntuación media de 413 . Se sitúa 80 puntos por debajo del promedio de 493 puntos de la OCDE, lo que indica que a los 15 años los estudiantes colombianos van rezagados, respecto a los estudiantes de un país que se sitúe en la media del desempeño de la OCDE como es el Reino Unido, del equivalente de dos años de escolaridad. Al citar el 
ranking PISA, la OCDE prefiere dar un intervalo limitado por el rango máximo y mínimo posible cuando hay alguna incertidumbre estadística. Así, Colombia ocupa el puesto 50-55 entre los sesenta y cinco países participantes. Por lo tanto, los estudiantes colombianos de 15 años obtienen peores resultados que los de Chile (449, puesto 44), Uruguay (426, puesto 46-50) y México (425, puesto 46-49), similares a los de Brasil (412, puesto 51-54), y significativamente mejores que los de Argentina (398, puesto 5559), Panamá (371, puesto 61-64) y Perú (370, también 61-64).

En lo relativo al sistema de educación superior, para comprender el estado de las universidades colombianas en el contexto de globalización actual, es absolutamente imprescindible seguir revisando el sistema de educación básica, secundaria y media del país ya que "el acceso a la educación superior depende de las condiciones de logro educativo de los niveles anteriores" (Sánchez Torres et al., 2002, p. 46). Para ello, nada mejor que poner de relieve los interesantes datos de un estudio llevado a cabo recientemente por investigadores colombianos (García Villegas et al., 2013) sobre la educación primaria, media y secundaria del país, quienes relevan que:

- históricamente, los colegios privados han obtenido mejores resultados en las pruebas SABER (pruebas de Estado que tienen lugar a lo largo de la educación primaria, secundaria y media) que los colegios públicos

- las instituciones que ofrecen educación gratuita en Colombia tienen los promedios más bajos de las pruebas SABER 
- los estudiantes con mayor capacidad de pago asisten a colegios con matrículas altas, y son estas instituciones las que cuentan con mejores promedios en las pruebas SABER

- los padres con mayor educación y, por lo tanto, con más dinero, son quienes tienen acceso a planteles de buena calidad y, por esa razón, el acceso a la educación de buena calidad termina estando restringido para los hogares menos favorecidos

- el nivel socioeconómico es más determinante en el rendimiento en idiomas que en el rendimiento en matemáticas y ciencias (los ricos pueden acceder a cursos de idiomas y/o viajar)

- hay una fuerte separación, incluso geográfica, entre, por un lado, los estudiantes de clase baja y media-baja que estudian en colegios públicos y que tienen al final un menor número de años de educación y peores rendimientos académicos, y, por el otro, los estudiantes de clase media-alta y alta que estudian en colegios privados y que tienen mayores años de educación y mejor desempeño en las pruebas oficiales

- no hay una relación estadísticamente significativa que sugiera una relación entre conflicto armado y calidad de la educación

- la pobreza y la desigualdad del municipio tienen un impacto negativo en la calidad de la educación: a mayor pobreza y desigualdad en un municipio, menor la calidad de la educación

- los estudiantes que viven en zonas urbanas obtienen, en promedio, 4 puntos más en las pruebas SABER que aquellos que viven en zonas rurales 
- el sistema educativo separa a las personas por razones de clase; los ricos estudian con los ricos en colegios privados, y los pobres con los pobres en colegios públicos: el 93\% de los estudiantes de estrato $1^{11}$ asisten a colegios públicos, mientras que el $98 \%$ de los estudiantes de estrato 6 asisten a colegios privados

- no solo se separa, sino que se tiene acceso a una educación desigual: mientras que un estudiante promedio de estrato 1 que asiste a un colegio público obtiene un puntaje de 43,14 en las pruebas SABER, un estudiante promedio de estrato 6 que asiste a un colegio privado obtiene un puntaje de 60,45/100. La diferencia es de 17 puntos

- mientras la diferencia entre jóvenes de estrato 1 y estrato 6 en 2008 era de 7 puntos, en 2011 fue de 17. Esto significa que de 2008 a 2011 se amplió la brecha entre estudiantes ricos y pobres en materia de desempeño escolar. En términos relativos, los primeros mejoraron su desempeño en 9 puntos, mientras que los segundos lo redujeron en 1 punto.

Estos problemas no son nuevos y numerosos analistas e investigadores los vienen señalando desde hace décadas. Es más, a pesar de que hay que reconocer que la cobertura se ha mejorado en términos globales (García Villegas et al., 2013 si hoy el sistema de educación aún sigue en crisis, se debe a que las medidas que se han venido tomando en los últimos treinta años son inapropiadas o insuficientes. Gracias a investigaciones como la antes citada, se puede entender mejor el porqué del bajo desempeño de los estudiantes colombianos en las diferentes pruebas internacionales. 
Hay en la actualidad, curso 2013-2014, 82 universidades de las cuales 50 son públicas y 32 privadas $^{12}$, repartidas en 236 campus a los que hay que añadir 182 sedes no presenciales y especiales. Los centros universitarios suman 1.030, de los cuales 481 son institutos y 47 hospitales universitarios; existen 29 escuelas doctorales (RD/2011 del 28 de enero). Teniendo en cuenta el número de universidades con relación a la población, por cada millón de habitantes hay 1,75 universidades, lo que es una ratio parecida al del Reino Unido. Las tres Comunidades autónomas que presentan mayor número de centros son, en este orden Madrid, Andalucía y Cataluña con respectivamente 156, 151 y 150. Madrid es la comunidad con mayor número de universidades privadas, 9 , lo que representa dos veces más que las públicas que son 6. Le sigue Cataluña con 5 ante 7 que son públicas, lo que representa casi la mitad. Andalucía cuenta con solo 1, mientras que las otras 10 son públicas.

España es uno de los países de la Unión europea que menos gasta en universidades, ya que solo les destinó, en 2012, el 0,89 \% del PIB, lo que ya representó una bajada del $-8,9 \%$ con relación al año anterior de 2011. Según el Instituto Nacional de Estadística, el gasto de I+D fue en 2012 de 13.391,6 m€ (lo que representó una disminución del -5,6 \% en relación al año anterior de 2011): 72,5 m€ se destinaron ${ }^{13}$ a otorgar 1.018 ayudas a la Formación del Personal Investigador (FPI), mientras que 54,2m€ se destinaron a otorgar 810 ayudas a la Formación del Profesorado Universitario (FPU) y 309,4m€ a proyectos de investigación. Las primeras, FPI, se otorgaron mayoritariamente a los sectores de la Tecnología y de las Comunicaciones (el 54,2\% de los destinatarios eran mujeres), las segundas a Ciencias Sociales y Humanidades (el 29,7 \% de los destinatarios eran mujeres). Comprobamos por lo tanto el trato diferenciado que reciben las llamadas ciencias (tecnología) y las humanidades y, por otra parte, se pone

\section{La "lenta desaceleración" de lo público en la Universidad}

\section{España: \\ Privatización de \\ la enseñanza \\ superior $\mathbf{y}$ aumento de la precariedad del cuerpo docente}


en evidencia nuevamente dónde se sitúa la feminización universitaria. Se otorgaron 294 ayudas Ramón y Cajal, Juan de La Cierva y otras del mismo tipo (el 42,4 \% fue atribuido a mujeres). Se dieron 3.176 proyectos de investigación fundamental no orientada, lo que representó 309,4m€ de los cuales 2.356m€ fueron a la Universidad. Casi la mitad del gasto se fue a Cataluña y Madrid con el 22,8 \% (536m€) y el 21,4 \% (505m€) respectivamente (Andalucía se llevó el 13,85, con 312m€). Comprobamos, por lo tanto, cómo desde hace algunos años, el presupuesto que se les asigna a las Universidades disminuye año tras año, lo que también pone en evidencia el propio Ministerio en sus informes.

En cuanto al número de estudiantes, habida cuenta de la bajada de la demografía observada en un $8 \%$, se calcula que habrá 1.438 .115 estudiantes. La inmensa mayoría son españoles, tan solo el $2 \%$ es extranjero, porcentaje que sigue bajando en relación a años anteriores. Los extranjeros representan la cuarta parte de los estudiantes de máster (exactamente el 27,5\%), de los cuales casi la mitad (40,9\%) procede de América latina y del Caribe. Como era de esperar, un dato interesante surge probablemente con la crisis, ya que, en las universidades públicas, para el curso 2013-2014, tanto el número de personal funcionario como contratado sufrió una disminución de $-5,1 \%$ y de $-1,2 \%$ respectivamente. Otro dato, si cabe más interesante aún, es el número casi idéntico de funcionarios y de contratados (48.423 y 46.884 respectivamente).

Ciertamente la mujer, como ocurre en otros ámbitos, ha llegado al cuerpo docente del alma mater, aunque, como era de esperar, su presencia es mayor en el cuerpo de los profesores titulares, sean estos de escuela universitaria (TEU) o de universidad (TU) y menor en el cuerpo de los 
profesores catedráticos, tanto de escuela universitaria (CEU) como de universidad (TU) con respectivamente el $2 \%$ y el $13 \%$ respectivamente. Recordemos que las propuestas de Rajoy y de Wert giraban en torno a cinco ejes.

a) El personal docente e investigador: para llegar a un $49 \%$ de personal docente contratado por los campus con la figura del "ternure track" captando así el talento internacional. b) La gobernanza con un Consejo universitario formado por entre 21 y 25 miembros de los cuales el $50 \%$ procedería del claustro de profesores, el $25 \%$ sería designado por las comunidades autónomas y el otro $25 \%$ estaría constituido por personal de reconocido prestigio internacional; el/la rector/a no procedería necesariamente del cuerpo docente. c) La financiación: los resultados de la investigación y por lo tanto del ranking aportarían a las universidades entre el $20 \%$ y el $25 \%$ de su presupuesto, los fondos privados deberían aumentarse. d) La diversidad y la especialización: repensar las titulaciones (eliminando aquellas con un número bajo de estudiantes) según las líneas de investigación teniendo en cuenta, no obstante, la accesibilidad territorial; los campus definirían sus líneas de docencia y de investigación prioritarias. e) La endogamia: estudiar nuevamente por qué el personal docente que obtiene la titularidad en la misma universidad en que se doctoró tiene más o menos méritos que los demás candidatos que se presentan a las plazas. Dicho de otra forma, se trata de cuestionar la presuposición según la cual la movilidad favorece la carrera. 
En Colombia se hace referencia al término "educación superior" para abarcar toda la educación postsecundaria formal y que según la OCDE (2013) en realidad corresponde a la "educación terciaria". Según esta organización, tradicionalmente, el término educación superior se refiere solo a la educación académica que conduce a cualificaciones de pregrado, y se considera un subgrupo de la educación terciaria, que también abarca otras formas de educación que conducen a cualificaciones por encima del nivel de educación secundaria, como la educación profesional y educación técnica.

De acuerdo con la Ley 30 de 1992, la educación superior a nivel de pregrado está compuesta por tres niveles de formación, que corresponden al técnico, al tecnológico y al profesional. Los programas académicos para estos tres niveles son ofrecidos por instituciones técnicas profesionales, instituciones universitarias o escuelas tecnológicas y universidades. En general, las modalidades de enseñanza ofrecidas son de tipo presencial, aunque algunas instituciones también ofrecen programas bajo la modalidad de educación a distancia. Así, existen cuatro tipos de instituciones de educación superior en Colombia:

- Universidades. Ofrecen programas académicos de pregrado y programas de posgrado que conducen a maestría y doctorado, y participan en la investigación científica y tecnológica.

- Instituciones universitarias. Ofrecen programas de pregrado hasta el nivel de título profesional y un tipo de programa de posgrado llamado “especialización” (un nivel de cualificación relacionado con la carrera, superior al pregrado, pero por debajo de la maestría).

\section{Colombia: Crisis en el sistema de financiación de las universidades estatales}


- Instituciones tecnológicas. Ofrecen programas hasta nivel tecnológico (se distinguen del técnico profesional por su base científica), y puede seguir hasta el título profesional siempre y cuando los programas en cuestión sean impartidos como ciclos propedéuticos.

- Instituciones técnicas profesionales. Ofrecen programas de pregrado en los niveles técnico/profesional para determinados trabajos o carreras.

Tabla 1

Tipos de Instituciones de Educación Superior en Colombia

Público 2011

Privado 2011

(cambios desde 2007) (cambios desde 2007)
Total 2011

(cambios desde 2007)

\begin{tabular}{lccc} 
Universidades & 32 (ningún cambio) & $48(+4)$ & $80(+4)$ \\
$\begin{array}{l}\text { Instituciones } \\
\text { universitarias }\end{array}$ & $27(-4)$ & $88(+16)$ & $54(-5)$ \\
$\begin{array}{l}\text { Instituciones } \\
\text { tecnológicas }\end{array}$ & $12(-4)$ & $42(-1)$ & $39(-10)$ \\
$\begin{array}{l}\text { Instituciones técnicas } \\
\text { profesionales }\end{array}$ & $9(-2)$ & $30(-8)$ & $288(+9)$ \\
\hline
\end{tabular}

Fuente: Informe preliminar (Ministerio de Educación Nacional, 2011) citado por varios autores (2013). 
Esta tabla resume perfectamente la tendencia de la educación superior colombiana en los últimos 10 años: aumento de la oferta de educación privada y debilitamiento de la educación pública, fenómeno que tiene lugar igualmente en España, como se ha visto anteriormente. En este sentido, Melo et al. (2014) recuerdan que mientras los recursos de las entidades privadas provienen esencialmente del cobro de derechos de matrícula, los de las instituciones de carácter público se originan en gran parte en transferencias del Gobierno Central y/o de los gobiernos sub-nacionales. En el periodo 2000-2012, el gasto estatal en este nivel de enseñanza representó en promedio 0,93\% del PIB, de los cuales la mitad corresponde a aportes de la Nación y el resto a recursos territoriales y a rentas parafiscales. Los aportes de la Nación están asignados principalmente al financiamiento de las Universidades Nacional, de Antioquia y del Valle que reciben alrededor del $30 \%$, el $10 \%$ y el $9 \%$ de los recursos, respectivamente. Según Melo et al. (2014), durante la última década los recursos estatales para educación superior no han mostrado cambios significativos respecto al PIB, lo cual sugiere que el Estado no ha respondido de forma paralela al crecimiento de la demanda por cupos de educación superior. Estos y otros problemas son abordados en la obra Desfinanciamiento de la educación superior en Colombia. La realidad de la crisis en el sistema de financiación de las universidades estatales (2012), publicada por el Consejo Nacional de Rectores Sistema Universitario Estatal.

En cuanto a la cobertura, es importante resaltar la prioridad que desde los años treinta del siglo pasado se otorgó a la educación superior con el fin de responder al proceso de urbanización y a las necesidades de desarrollo del país. A pesar del esfuerzo de varios gobiernos por ampliar el acceso de la 
población a este nivel de formación académica, la tasa de cobertura apenas ascendió de 3,9 \% en 1970 a 8,9 \% en 1980 y a 13,4 \% en 1990. Durante los últimos 20 años, el acceso a la educación superior aumentó a un mayor ritmo, lo que se tradujo en una tasa de cobertura bruta de $24,0 \%$ en el año 2000 y de $42,4 \%$ al final de 2012. Durante este periodo, la población matriculada a nivel de pregrado ascendió de 487.448 estudiantes en 1990 a 1.841.282 en 2012, lo que significó una ampliación de los cupos cercana a $278 \%$. Vale la pena señalar que gran parte del aumento reciente del número de matriculados tiene origen en aumento de cupos para educación técnica y tecnológica, que pasaron de 183.319 en 2002 a 622.746 en 2012.

Este incremento refleja la política de educación superior durante la última década, la cual dio prioridad a este tipo de formación. En efecto, mientras la tasa de cobertura en educación técnica y tecnológica ascendió de 4,8 \% en 2002 a 14,3 \% en 2012, en el nivel profesional esta tasa aumentó de 19,6 $\%$ en el primer año a $28,1 \%$ en el último.

Adicionalmente, Melo et al. (2014) nos informan de que durante las dos últimas décadas la cobertura en educación superior ha registrado adelantos importantes, especialmente en la formación técnica y tecnológica. No obstante, desde una perspectiva internacional, la tasa de cobertura del país es relativamente baja (42,4 \% para el 2012), cuando se compara con países desarrollados y con países de América Latina como Argentina, Chile, Cuba, Uruguay y Puerto Rico. También se puede destacar el aumento de 844.594 estudiantes matriculados en pregrado entre 2003 y 2012, lo cual significó un crecimiento cercano al $85 \%$ entre estos dos años. La variación en el número de estudiantes matriculados se explica en gran parte por el SENA $^{14}$ (Servicio Nacional de Aprendizaje), con el $35 \%$, y por la 
Universidad Abierta y a Distancia con el $5 \%$. Esta última institución contaba hasta 2014 con el mayor número de estudiantes matriculados.

Aunque ha habido mejoras en la cobertura, esta no se transformará en una mejora de la educación si no hay toda una política que la acompañe, ya que de nada sirve aumentar los cupos en los establecimientos educativos del país si no hay recursos para acoger y formar correctamente a la población. En efecto, como se estableció anteriormente, a la universidad colombiana ingresan principalmente las élites de los colegios privados y públicos. El sistema deja al margen a los ciudadanos de estratos pobres y de zonas rurales, quienes son los más excluidos del acceso a la educación superior y con mayores dificultades para endeudarse a fin de pagar sus estudios. Por ende, este es el grupo social hacia el cual debe focalizarse la política gubernamental de "cobertura con equidad” (Gómez \& Celis, 2009, p. 113).

Respecto a los docentes, de acuerdo con el Ministerio de Educación Nacional, en el año 2012 el sistema de educación superior contaba con 111.124 profesores, de los cuales 33.084 laboraban con un contrato de tiempo completo, 12.767 bajo la modalidad de medio tiempo y 65.273 con un esquema de hora cátedra. Por nivel de formación, el 40,5\% de estos docentes tenía título de pregrado, el 32,0 \% de especialización, el 22,3\% de magister y el 5,2 \% de doctorado. Llama la atención el alto número de profesores contratados por horas $(58,7 \%)$ y el bajo porcentaje de docentes con doctorado (5,2\%), que además están concentrados en pocas universidades. Según la OCDE, esta situación puede estar afectando las actividades de investigación, la formación académica de los estudiantes y en general los resultados del sistema educativo. 
Tabla 2

Personal docente de las instituciones de educación superior y sus respectivas cualificaciones 2002-2009

\begin{tabular}{|c|c|c|c|c|c|c|c|c|c|c|}
\hline Máxima cualificación & 2002 & $\begin{array}{l}2002 \\
(\%)\end{array}$ & 2003 & 2004 & 2005 & 2006 & 2007 & 2008 & 2009 & $\begin{array}{c}2009 \\
(\%)\end{array}$ \\
\hline Universitario & 39.063 & $47 \%$ & 38.985 & 38.597 & 39.265 & 39.616 & 42.929 & 46.555 & 46.741 & $42 \%$ \\
\hline Especialización & 27.420 & $33 \%$ & 33.244 & 33.760 & 36.221 & 37.979 & 36.406 & 37.958 & 38.076 & $34 \%$ \\
\hline Maestría & 14.414 & $17 \%$ & 15.457 & 17.309 & 19.657 & 19.471 & 19.288 & 21.026 & 21.093 & $19 \%$ \\
\hline Doctorado & 2.445 & $3 \%$ & 2.617 & 2.871 & 3.193 & 3.540 & 3.522 & 4.102 & 4.578 & $4 \%$ \\
\hline Total & 83.342 & $100 \%$ & 90.303 & 92.537 & 98.336 & 100.606 & 102.145 & 109.644 & 110.788 & $100 \%$ \\
\hline
\end{tabular}

Fuente: Informe preliminar (Ministerio de Educación Nacional, 2011) citado por varios autores (2013).

Aunque estas cifras no son muy actuales, son las más recientes a las que tuvimos acceso; en ellas se puede ver que los doctores en las universidades públicas son una pequeña minoría, aunque en 2015 es muy probable que la cifra esté en más del $5 \%$ ya que en los últimos diez años muchos colombianos han obtenido doctorados en el extranjero y regresado a ejercer la docencia en el país. Este regreso ha sido incitado por diversas políticas gubernamentales, de las cuales resaltan dos. La primera, es la financiación total o parcial de estudios de posgrado en Colombia o en el extranjero, al cabo de los cuales los beneficiarios deben regresar a trabajar al país. El otro programa oficial se bautizó "Es hora de volver" y su objetivo es, como su nombre lo indica, que los cerebros fugados retornen al país para trabajar 
en empresas y en universidades. No obstante, a pesar de los cantos de sirena del gobierno colombiano hacia los doctores expatriados, muchos han decidido no volver tras ver las inconsistencias del programa y la falta de cumplimiento del gobierno y de las universidades.

Como se ha ido observando en los últimos años y como así lo dijo Mariano Rajoy al llegar al poder, las tasas universitarias debían aumentar. Recordemos que una de las propuestas del Gobierno fue disminuir el número de becas y aumentar las tasas universitarias, cosa que hizo. No se hablaba de democratización, sino de transformación de la Universidad mediante la competitividad. Esta se traduce por dinero: a) dinero por alumno para que las universidades compitan entre ellas para atraerlos y b) dinero por actividad científica (recompensas) para que los profesores compitan entre ellos buscando proyectos de investigación a gran escala y publicando los resultados (los famosos rankings o las revistas de rango A, en las que el factor primordial es el impacto). El cuadro número 3 nos plasma una fotografía en la que aparece el precio por crédito en las tres comunidades mencionadas, Madrid, Cataluña y Andalucía, a las que hemos añadido Galicia que es la comunidad en la que los precios son los más baratos. Se indican los precios por crédito sin repetición de matrícula (el crédito se encarece cuando se repite) Se puede observar, como ha venido siendo en los últimos años, que los precios se disparan en los estudios de máster no habilitantes llegando a alcanzar los $65 €$ por crédito en Madrid y Cataluña. De ahí que los estudiantes lleguen a pagar $3.000 €$ o más por un máster que, según algunos, les abrirá las puertas de la vida profesional futura. 
Tabla 3

Precio del crédito en diferentes universidades según el grado de matrícula. Media de 60 créditos: $1.105 €$

Grado $\begin{gathered}\text { Máster } \\ \text { habilitante }\end{gathered} \begin{gathered}\text { No } \\ \text { habilitantes }\end{gathered} \begin{gathered}\text { Doctorado } \\ \text { tutela }\end{gathered} \begin{gathered}\text { Doctorado } \\ \text { examen }\end{gathered}$

\begin{tabular}{lccccc}
\hline Andalucía & $12,62 €$ & $19,50 €$ & $41,50 €$ & $60,30 €$ & $127,90 €$ \\
Cataluña & $33,52 €$ & $40,88 €$ & $65,41 €$ & $398,33 €$ & $155,78 €$ \\
Galicia & $11,89 €$ & $25,03 €$ & $26,49 €$ & $200 €$ & $117,09 €$ \\
Madrid & $20,30 €$ & $42,75 €$ & $65 €$ & $390 €$ & $143,15 €$ \\
\hline
\end{tabular}

Fuente: Ministerio de Educación, Cultura y Deporte (2013). Recuperado de: www.mecd.gob.es

Es interesante mencionar respeto a las tasas académicas, la relación existente entre, por una parte, la tasa de rendimiento según si la universidad es pública o privada y, por otra parte, según se trate de grado o de máster. En el primer caso, el porcentaje de créditos superados en las formaciones de grado es mayor en las universidades privadas que en las públicas $(84,5$ $\%$ y 70,2 \% respectivamente) con una diferencia de casi 15 puntos. En cuanto al porcentaje en las formaciones de máster, es mayor, aunque solo de un punto, en las públicas que en las privadas (respectivamente 87,8 \% y $86,3 \%$ ). Tanto en un caso como en otro, los estudiantes presentan un mejor rendimiento (en otras palabras, aprueban) en los estudios de máster (comparándolos con los del grado), aunque hay una diferencia neta en las universidades públicas, en donde el porcentaje llega a casi el $90 \%$ (concretamente al 87,8\%), mientras que en las formaciones de grado llega a un poco más de los dos tercios, concretamente al 70,2\%. 
¿Cómo se pueden interpretar estos datos? Se pueden barajar varias hipótesis. Una de ellas que consistiría en decir que en las Universidades privadas los estudiantes "compran" los títulos, de ahí que el rendimiento sea mayor, sobre todo en los grados. Otra interpretación consistiría en decir que los estudiantes de las universidades privadas, cuya mayor parte procede de centros de enseñanza secundaria privados o concertados, tiene mejores rendimientos porque al no trabajar pueden dedicarse más tiempo al estudio, aunque para afirmar esto, tendríamos que compararlo con datos no disponibles.

Esta diferencia de rendimiento entre el grado y el máster se da en las cuatro comunidades autónomas mencionadas. Los mejores rendimientos los obtienen los estudiantes de Cataluña $(91,1 \%)$, pisándoles los talones los de Galicia (90,8 \%), los menores rendimientos en los grados los obtienen los estudiantes de Andalucía (69,2 \%), seguidos por los de Galicia (71,5 $\%$ ). Para resumir, los estudiantes de las universidades privadas consiguen mayor rendimiento que los de las públicas en cuanto al grado, pero luego esta tendencia se inversa. El mayor rendimiento en los estudios de máster podría explicarse por el hecho de que el estudiante ya no toma los estudios de máster como una continuidad de los estudios de secundaria, como lo hace probablemente con los de grado, sino como una especialización para lo que busca aquel máster que mejor se adapte a su proyecto profesional y que le pueda ofrecer mayores posibilidades de empleo. El aumento de tasas universitarias puede que aumente en rendimiento, pero disminuye la equidad. La equidad también disminuye cuando las becas concedidas se reducen: durante el curso 2012-2013, el número de becas concedidas disminuyó en unas 10.000 mientras que las solicitudes aumentaron en 30.000 (cf. Parellada ${ }^{15}$ ). 
En su informe de 2013, la OCDE indica que:

Además de ayudar a jóvenes a acceder a unos estudios de educación superior que de otro modo no podrían costearse, los créditos del ICETEX (Instituto Colombiano para la Educación en el Exterior) también contribuyen a reducir los índices de deserción de los beneficiarios, tal y como se muestra en el siguiente apartado (...)

El equipo de análisis valora muy positivamente el sistema del ICETEX, que goza de una buena reputación de liderazgo internacional en el ámbito de los créditos educativos. Sus objetivos son de una enorme solidez, ha contribuido de manera muy notable al acceso y a la equidad y el programa se ejecuta con gran eficiencia (...) (p. 121).

Esto es normal dado que una de las "recomendaciones" de esta organización es que haya cada vez menos recursos públicos en la financiación de la educación pública. Esta exigencia fue una de las principales causas de la crisis que hubo en 2011 en Colombia, por la cual se paralizaron las universidades públicas durante varios meses. Las asociaciones de estudiantes sostenían que:

La política financiera del Banco Mundial se aplica a costa de desfinanciar a la Universidad Pública, tan es así, que los recursos institucionales del ICETEX como porcentaje del total de las universidades, pasó del 7,4 \% en el 2000 al 20,1 \% en 2010, mientras que las transferencias nacionales a las universidades pasaron del $82,6 \%$ al $59,4 \%$ en igual periodo. Todo esto, mientras desembolsa
Colombia: Más cobertura con iguales (o menos) recursos 
un préstamo por US $\$ 500$ millones destinados a los créditos estudiantiles, actuaciones que han sido rechazadas por múltiples actores de la comunidad educativa. Mesa Amplia Nacional Estudiantil (MANE, 2013). ${ }^{16}$

Al final, la presión de la comunidad en su conjunto logró frenar el proyecto de ley que pretendía dar vía libre a la creación de más instituciones universitarias privadas en el país.

Por todo lo expuesto hasta aquí, resulta evidente que, a la universidad colombiana, pública o privada, ingresan principalmente, por un lado, los jóvenes de clase alta y media alta, quienes son formados en los mejores colegios privados de las grandes ciudades y, por otro, los mejores estudiantes de los colegios públicos. Queda pues excluida la mayor parte de los campesinos, los indígenas y los afro-descendientes (Ortiz \& Guzmán, 2008), es decir, la población que habita en las zonas más hostiles e inhóspitas del país. Históricamente, estos pueblos han vivido en la periferia del mismo y sufrido el olvido del Estado, la violencia de la guerra y el racismo e indiferencia de muchos de sus compatriotas.

En términos de matrículas, el acceso a la universidad depende de diversos factores, principalmente, del tipo de universidad y del estrato socioeconómico del estudiante. A las universidades públicas ingresa aproximadamente el $10 \%$ de los aspirantes y el coste de la matrícula varía en función de los ingresos de los padres o acudientes del estudiante. Para los de estratos 1 y 2, la matrícula es asequible. En cuanto a las universidades privadas, los cupos varían de universidad en universidad y las matrículas comienzan aproximadamente en mil euros por semestre y 
pueden alcanzar más de seis mil. Por esta razón, los estudiantes más pobres solo pueden estudiar en estos establecimientos si tienen becas de excelencia (completas).

Muchos estudiantes que no pueden ingresar en las universidades (públicas o privadas) porque su nivel académico y/o económico no se lo permite, deben dirigirse a las instituciones técnicas y tecnológicas públicas y privadas, que son a menudo caras y de baja calidad.

En lo relativo a las tasas de deserción, los indicadores revelan una situación preocupante. En efecto, de acuerdo con un estudio del Ministerio de Educación Nacional en el cual se hace seguimiento a las tasas de deserción por cohortes, se concluye que en promedio uno de cada dos estudiantes no culmina sus estudios superiores (Ministerio de Educación Nacional, 2009). En el nivel técnico, la tasa de deserción, que es acumulativa, alcanza 33,4 $\%$ en el primer semestre y 71,1\% en el décimo. Así mismo, en el nivel universitario la tasa de deserción asciende de 18,6 \% en el primer semestre a 47,0 \% en el décimo. Cuando se evalúa la permanencia de los estudiantes por áreas del conocimiento, según el informe de la OCDE, las mayores tasas de deserción se observan en agronomía, veterinaria y afines, y en ingeniería, arquitectura y afines, con unas tasas promedio acumuladas al finalizar el décimo semestre de 57,7 \% y de 56,3\%, respectivamente. $\mathrm{La}$ menor tasa, por el contario, se registra en ciencias de la salud con 40,1\% acumulado en el décimo semestre. En general, las razones por las cuales los estudiantes abandonan sus estudios, de acuerdo con el estudio del Ministerio de Educación Nacional (2009), son principalmente de índole personal (edad, género, situación laboral), académicas (tasa de repetición, área de conocimiento y puntaje en las pruebas SABER), socioeconómicas (nivel de ingresos, educación de la madre, tasa de desempleo de la región, 
número y posición entre los hermanos) e institucionales (facilidad de apoyo financiero).

Según la OCDE, los motivos de la deserción en Colombia suelen ser de índole económico-financiera, académica, institucional o personal. Por eso, uno de los objetivos importantes de los créditos ACCES $^{17}$ del ICETEX es reducir la deserción eliminando o minimizando los motivos económicos subyacentes. Según este organismo, el programa de créditos ha resultado "bastante efectivo" a la hora de reducir los niveles de deserción y mejorar las probabilidades de completar los estudios de los estudiantes en situación de riesgo. Citan los datos del SPADIES ${ }^{18}$, que mostraron que, globalmente, los estudiantes con créditos ACCES tenían una tasa de deserción por cohorte del $35.6 \%$, mientras que los que carecían de créditos tenían una tasa de deserción del $52.1 \%$.

Sin embargo, sobre este tema Gómez Campo y Celis Giraldo (2009) apuntan que para las Instituciones de Educación Superior las deficiencias académicas y las restricciones económicas son los factores que generan el rezago académico y la posterior deserción de la mayoría de estudiantes que ingresan en la educación superior, en especial, de los provenientes de los estratos 1 y 2. Para ellos, los créditos no son en absoluto suficientes ya que debe haber también una política de acompañamiento integral.

A pesar del reconocimiento de las grandes insuficiencias en conocimientos y competencias académicas en los jóvenes de menores estratos, aspirantes a educación superior, la mayoría de las instituciones no cuenta con acciones afirmativas (Económicas, Académicas, Subsidios de sostenimiento, Sicosociales) orientadas a elevar y equilibrar, desde el nivel 
medio, su capacidad de ingreso en la educación superior. Las pocas que promueven programas de admisión especial de estratos 1 y 2 y grupos étnicos son en su mayoría públicas: las Universidades de Antioquia, de Caldas y Nacional de Colombia y el Instituto Tecnológico Metropolitano de Medellín, que tienen como población beneficiaria a los jóvenes procedentes de comunidades indígenas, de municipios pobres y de estratos 1 y 2. Como resultado de esta articulación, varios egresados de estos colegios han podido acceder tanto al Tecnológico Metropolitano de Medellín, como a otras universidades locales como la Universidad Nacional y la de Antioquia. Antes de esta articulación, señalan Gómez Campo y Celis Giraldo (2009), pocos egresados de estos colegios podían aspirar a ingresar en el nivel superior.

Finalmente, los resultados de un estudio realizado por Melo et al. (2014) sobre la eficiencia de las universidades, reafirman la importancia de las variables socioeconómicas en el logro académico de los estudiantes de educación superior. Esto demuestra que, aunque muchas instituciones educativas tienen margen para mejorar sus niveles de eficiencia, están restringidas por la influencia de los factores de entorno de sus estudiantes. Así, para lograr un mejoramiento de los resultados académicos, sugieren los autores de la investigación, las medidas de política del Estado y las estrategias de las instituciones deben tomar en cuenta además de los criterios en la contratación de docentes, la definición de incentivos para la investigación y los aspectos administrativos y financieros, los mecanismos que permitan ayudar a contrarrestar el impacto negativo derivado de las condiciones socioeconómicos de los estudiantes y de otros factores ambientales. 
Siempre según datos del Ministerio, el índice de paro juvenil en España es 3 veces mayor al resto de los países de la OCDE, incluso antes de la crisis. A los jóvenes les cuesta entrar en el mercado de trabajo, en España este factor toma mayor importancia que en otros países. Pero, ¿se debe esta entrada tardía solo a la falta de experiencia o se debe también a otros factores. Seguramente a varios factores y no solo a uno. La falta de conocimientos del mundo laboral y el funcionamiento de las empresas es ciertamente uno de ellos, pero no el único. La crisis ha incrementado el índice de paro en 10 puntos para los jóvenes que tienen estudios universitarios, pasando de un índice de 5,4\% en 2007 al 15.2 \% en 2012; también se ha incrementado en aquellos jóvenes doctores, aunque en un porcentaje menor, pasando de un índice del 2,7 \% en 2007 al 4,7 \% en 2012. Los egresados no obtienen mejor salario, aunque sí sufren menos del paro que los jóvenes que no tienen estudios o solo estudios secundarios. En resumen, tener estudios universitarios no conlleva en sí mismo obtener un trabajo, aunque sí está correlacionado con el riesgo menor de sufrir del paro. Este no es un problema de la Universidad, es un problema del mercado laboral español que es estructural independientemente de la crisis, ya que España es uno de los países en los cuales se agudiza la dificultad de crear empleo.

Las "políticas de ajuste estructural" impuestas alrededor del mundo por organizaciones político-financieras como el Banco Mundial, la OCDE y el FMI, los tratados de "libre comercio", el desarrollo de las tecnologías de la comunicación y la política económica basada en el "crecimiento" han obligado a muchos gobiernos a reestructurar sus sistemas de educación a fin de hacerlos rentables y más competitivos en el contexto del mundo globalizado actual, el cual gira en torno a la economía, los servicios y las

\section{España y \\ Colombia en la era de la "economía del conocimiento"}

\section{España: La tan traída y llevada empleabilidad de los estudiantes universitarios}

\section{Colombia: Crece la economía a la par de la privatización de la educación}


finanzas. Estos cambios en las políticas económicas y educativas han provocado que la atención y los esfuerzos de los gobiernos se centren en la relación existente entre la educación, el capital humano y la producción de conocimiento. Por ende, dicha relación se ha vuelto imprescindible para todo país que desee aumentar su crecimiento económico, su productividad y su participación en la "economía del conocimiento". Colombia está directamente concernida por esta realidad ya que es un país que desde hace décadas viene reformando su sistema escolar en función de las demandas de los organismos internacionales antes citados, como lo señala Cruz Atehortúa (2012):

Existe real coincidencia entre las políticas educativas trazadas por el Banco Mundial y las recientes políticas para la educación superior diseñadas en Colombia. El enfoque mercantilizador, articulado a las necesidades y demandas del sistema económico, se expresa en concepciones utilitaristas visibles en los Planes Nacionales de Desarrollo y fortalece las tendencias privatizadoras de la educación pública (p. 19).

Mientras en varios estudios sociológicos y económicos realizados recientemente sobre el sistema de educación en Colombia (Sánchez Torres et al. 2002; Ortiz \& Guzmán, 2008; Gómez Campo \& Celis Giraldo, 2009; Corzo et al., 2011; Cruz Atehortúa, 2012; Noreña Jaramillo \& Rincón Laverde, 2012; García Villegas et al., 2013; Melo et al., 2014), las palabras más recurrentes son desigualdad, rezago, brecha, ricos, pobres, colegios públicos, colegios privados, deserción, créditos, desfinanciamiento, regiones rurales, acciones afirmativas, formación ciudadana, racismo, etc., en los documentos de la OCDE (informes de 2013 y 2014) o del Banco Mundial (p.ej. Jaramillo, 2005), las reformas impuestas a Colombia giran 
en torno a un campo semántico e ideológico muy concreto: evaluación, internacionalización, competencias, eficiencia, indicadores, retos, gobernabilidad, etc. Dichos vocablos dejan claro que a través de sus "propuestas" de reforma, esas organizaciones "recomiendan" que Colombia entre en el sistema estandarizado de educación que se ha impuesto en el todo el mundo, empezando por los Estados Unidos. Por ende, es evidente que los documentos de la OCDE y del Banco Mundial tienen un enfoque puramente económico y utilitarista que deja completamente de lado la educación y el conocimiento vistos desde una perspectiva pedagógica, crítica y humanista. Así, Corzo et al. (2011) afirman que el producto de las reformas de la educación propuestas por estas organizaciones es un

Individuo visibilizado como fuerza de trabajo; un ciudadano pasivo que interviene de manera tímida, poco habituado a las prácticas políticas, pero muy acostumbrado a sentirse ciudadano en la medida que sus posibilidades de consumo se lo permiten (p. 209).

Por todo lo anterior, podemos concluir que los documentos de los organismos internacionales pasan por alto lo que promulga la Constitución colombiana en materia de educación, a saber, formar a ciudadanos críticos con base en una educación integral. 
Hemos puesto de manifiesto a lo largo del artículo cómo el neoliberalismo

Conclusiones

y la globalización han impuesto cambios radicales a nivel mundial en las misiones encomendadas a la Universidad. A pesar de que el acceso a ella, tanto en España como en Colombia, es mayor que en otros tiempos, la estratificación, sea esta social, de origen, de género o de recursos económicos, sigue estando vigente, e incluso se han incrementado las diferencias. Las universidades privadas son numerosas en ambos países y, por ende, a ellas acceden aquellos estudiantes cuyos padres tienen los recursos necesarios para pagarlas. Por otra parte, la Universidad ya ha dejado de ser el lugar por excelencia del conocimiento y de la investigación para convertirse, sobre todo, en un lugar de formación (docencia para el profesorado y aprendizaje a lo largo de toda la vida para el alumnado) que debe dar respuesta a necesidades precisas, no de la sociedad sino de organismos supranacionales como la OCDE, el FMI o la misma Unión Europea con el Plan Bolonia. El cambio de gobernanza consiste pues en profesionalizar, con cánones propios de las empresas, al conjunto del cuerpo docente universitario llevando las técnicas de gestión (de management) propias del sector privado a la Universidad.

"La educación puede ser la gran fecundadora o la gran esterilizante de los hombres, y los hombres pueden ser los grandes transformadores de los pueblos o los más formidables obstáculos para su progreso", sostenía el médico colombiano Héctor Abad Gómez hace cuarenta años (asesinado por promover ideas "revolucionarias"). Hoy en día, la idea de progreso que defienden los dirigentes en España y Colombia equivale al crecimiento económico y para llegar a este hay que ser competitivos, aunque haya que destruir a los conciudadanos (competencia) y acabar con el planeta. 
En el contexto de globalización y de crisis actual, es evidente que España y Colombia deben abrirse al mundo. En el país europeo el movimiento ciudadano de los indignados está haciendo mover el ajedrez político tradicional y en el suramericano, el gobierno y las guerrillas han mostrado interés en poner fin a una guerra de seis décadas. Ambos países tienen historias diferentes, pero comparten preocupaciones y realidades comunes. Por consiguiente, a los dos lados del Atlántico se deben formar estudiantes capaces de trabajar y competir de manera ética, justa, crítica y consciente en un mundo que cada vez tiene menos fronteras y en el que la brecha entre pobres y ricos es la más importante en la Historia de la Humanidad. No se trata, pues, de cerrarse al mundo ni de negarse a entrar en una globalización en la que ya estamos sumergidos. Empero, para que los niños y jóvenes puedan convertirse en verdaderos ciudadanos-profesionales que salgan a estudiar y a laborar a nivel local o internacional, primero se tienen que resolver los graves problemas de injustica social que aquejan a ambos países desde hace años, empezando por el acceso de las nuevas generaciones a una educación igualitaria y de calidad en todos los rincones de los dos países. Si los responsables políticos no asumen estos retos, el papel de las instituciones educativas se limitará exclusivamente a instruir y no a educar, como lo pretenden la OCDE, el Banco Mundial y hasta la Unión Europea; a capacitar a los estudiantes en el dominio de las competencias exigidas por una economía que está dirigida por el mercado (Giroux, 2005), lo cual traerá como consecuencia que ambas naciones se pierdan en las sombras de la desigualdad, del individualismo, del exilio y de la guerra, en el caso de Colombia. 
Corzo, A. I., Duque, J., Medina, L. \& Torres, S. (2011). “El impacto de las Referencias políticas neoliberales en América Latina. Un análisis de la educación en Perú, Colombia y México". Temas de Nuestra América. Revista de Estudios Latinoamericanos, 1, (50), pp. 189-221.

Cruz, A. L. (2012). "El Banco Mundial y las políticas educativas en Colombia". En Simposio Internacional de Pedagogía, Humanidades y Educación. Escuela y Pedagogía Transformadora. Abril 19 y 20 de 2012. Universidad Autónoma de Occidente, Cali, Colombia. Red Iberoamericana de Pedagogía.

Cruz, L. \& Sanz, L. (2010). "Endogamia, productividad y carreras universitarias". Documento de trabajo, Instituto de Políticas y bienes públicos, CSI, Madrid.

Fernández, M. (1999). "La transformación de la Universidad española". Revista Interuniversitaria de Formación del profesorado, 34, enero/abril, pp. 31-37.

Gaboriau, P. (2008). Le chercheur et la politique. L'ombre de nouveaux inquisiteurs. Montreuil: Editions Aux lieux d'être.

García, M., Espinosa, J. R., Jiménez, F. \& Parra, J. D. (2013). Separados y desiguales. Educación y clases sociales en Colombia. Bogotá: Centro de Estudios de Derecho, Justicia y Sociedad, Dejusticia, 2013.

Giroux, H. (2005). "War talk and the shredding of the social contract: Youth and the politics of domestic Militarization". En G. Fischman; P. McLaren; H. Sünker y C. Lankshear (Ed.) Critical theories, radical pedagogies, and global conflicts, pp. 52-68. Lanham: Rowman \& Littlefield.

Gómez, V. M. \& Celis, J. E. (2009). "Crédito educativo, acciones afirmativas y equidad social en la educación superior en Colombia". Revista de estudios sociales, 33, pp. 106-117. 
Jaramillo, I.C. (2005). "Internacionalización de la educación superior en Colombia". En De Wit, H., Jaramillo, I. C., Gacel-Avila, J., \& Knight, J. Educación Superior en América Latina. La dimensión internacional. Washington: Banco Mundial.

Lyotard, J. F. (1984). The postmodern condition: A report on knowledge [Vol. 10]. Minnesota: University of Minnesota Press.

Lyotard, J. F. (1992). The Postmodern Explained to Children Correspondence 1982-1985. Translation and edition J. Pefanis \& M. Thomas. Sydney: Power publications.

Lyotard, J. F. (1995). Foreword: Spaceship. En Education and the post modern condition. Peters, M.A. (Ed.), xi-xx. Westport: Bergin \& Garvey.

Marsi, L. (2010) "Universidad, liberalismo y economicismo a principios del siglo XXI”, en La Universidad por un mundo mejor. En Actas del Congreso Universidad 2010, 7mo Congreso Internacional de Educación Superior, X Taller Internacional "La educación superior y sus perspectivas" (memoria n. 055), Universidad de La Habana (Cuba), 8-12 febrero, pp. 364-374.

Melo, L. A., Ramos, J. E., \& Hernández, P. O. (2014). “La Educación Superior en Colombia: Situación actual y análisis de eficiencia”. Borradores de Economía (808). Bogotá: Banco de la República de Colombia.

Ministerio de Educación Nacional (2009). Deserción Estudiantil en la Educación Superior en Colombia. Ministerio de Educación Nacional de Colombia.

Ministerio de Educación Nacional (2011). Informe Preliminar sobre la Educación Superior en Colombia. Bogotá: Ministerio de Educación Nacional de la República de Colombia. 
Ministerio de Educación, Cultura y Deporte (2014). Datos básicos del Sistema universitario español. Curso 2013-2014. Madrid: Secretaría general de Universidades.

Montero, M. (2010). "El proceso de Bolonia y las nuevas competencias". Tejuelo, 9, pp. 19-37.

Noreña, F. \& Rincón, T. (coordinadores) (2012). Desfinanciamiento de la educación superior en Colombia. La realidad de la crisis en el sistema de financiación de las Universidades Estatales. Pereira: Sistema Universitario Estatal (SUE).

Núñez, C. E. (2013) Universidad y Ciencia en España. Claves de un fracaso y vías de solución. Madrid: Gadir.

Oppenheimer, A. (2010). ¡Basta de historias! La obsesión latinoamericana con el pasado y las doce claves del futuro. Madrid: Debolsillo.

Ortiz, J.A.C., \& Guzmán, E.C. (2008). “Indígenas y afrodescendientes en la universidad colombiana: nuevos sujetos, viejas estructuras". En Cuadernos interculturales, 6 (10), pp. 62-90.

Peters, M. (2005). "The Posthistorical University? Prospects for Alternative Globalizations”. En G. Fischman; Peter McLaren; H. Sünker \& C. Lankshear (Ed.). Critical theories, radical pedagogies, and global conflicts, pp. 69-86. Lanham: Rowman \& Littlefield.

Readings, B. (1996). The university in ruins. Boston: Harvard University Press.

Sánchez, A., \& Otero, A (2012) Educación y reproducción de la desigualdad en Colombia, in M. del P. Esguerra U., Reportes del emisor, (154), Bogotá: Banco de la República. 
Sánchez, F.J., Quiros, M., Reverón, C. \& Rodríguez, A. (2002) Equidad social en el acceso y permanencia en la universidad pública: determinantes y factores asociados. Universidad de los Andes, Centro de Estudios sobre Desarrollo Económico.

Varios autores (2013). Reviews of National Policies for Education: Tertiary Education in Colombia 2012. OCDE and the International Bank for Reconstruction and Development/The World Bank.

Varios autores. (2014). Education at a Glance. OCDE indicators. 
1. En el curso 1976-1977 las mujeres representaban el 39\% de la Notas población universitaria, diez años después igualaban a los hombres y en el curso 1996-1997 les superaban en un 6\% (cf. Fernández Enguita, 1999). Durante el curso 2012-2013, el porcentaje de mujeres estudiando en la Universidad es superior al de los hombres, lo que representa el 54,3\%. El porcentaje de mujeres egresadas también es superior al de los hombres, lo que representan el 57,6\%, con lo cual las mujeres no solo acceden más a los estudios universitarios que los hombres, sino que además rinden mejor (aprueban más) (cf. Ministerio de Educación, Cultura y Deporte, 2013.

2. http://goo.gl/VveMWT

3. El inicio fue en 1998 cuando en la Sorbona cuatro países lo firmaron.

4. Véase también el Foro IMHE Institutional Management in Higher Education.

5. A partir del puesto 200, aparecen las Universidades Autónoma de Madrid, Complutense, de Barcelona y de Valencia.

6. Véase Marsi (2010).

7. Las mareas blancas (de sanidad) y las verdes (de educación) son una muestra de ello porque lo que pretende el gobierno del Partido Popular es la privatización de la educación y de la sanidad. El Movimiento del 11M, llamado también de los Indignados o la Spanish Revolution debe situarse en este contexto. En la globalización basada en el neoliberalismo todo se privatiza, podemos añadir otros ejemplos como el de las eléctricas o el agua.

8. No podemos, en el marco del presente artículo, exponer los resultados de las elecciones europeas de 2014 y el de las municipales españolas de 2015, aunque sí podemos mencionar la caída de los dos grandes partidos políticos que se disputaban hasta entonces en las democracias la llamada alternancia. 
9. La última actualización se hizo el 29 de mayo de 2013.

10. http://www.mineducacion.gov.co/1621/w3-article-89266.html

11. La estratificación socio-económica es la clasificación de los inmuebles residenciales que deben recibir servicios públicos. Se realiza principalmente para cobrar de manera diferencial (por estratos) los servicios públicos domiciliarios permitiendo asignar subsidios y cobrar contribuciones. De esta manera, quienes tienen más capacidad económica pagan más por los servicios públicos y contribuyen para que los estratos bajos puedan pagar sus tarifas (...) De estos, los estratos 1, 2 y 3 corresponden a estratos bajos que albergan a los usuarios con menores recursos, los cuales son beneficiarios de subsidios en los servicios públicos domiciliarios; los estratos 5,y 6 corresponden a estratos altos que albergan a los usuarios con mayores recursos económicos, los cuales deben pagar sobrecostos (contribución) sobre el valor de los servicios públicos domiciliarios. El estrato 4 no es beneficiario de subsidios, ni debe pagar sobrecostos, paga exactamente el valor que la empresa defina como costo de prestación del servicio. Recuperado de http://es.wikipedia.org/wiki/Estratificaci\%C3\%B3n_socioecon $\% \mathrm{C}$ 3\%B3mica_en_Colombia

12. Todos los datos están sacados de www.mecd.gob.es [consultado el 28 de junio de 2015] y de Ministerio de Educación, Cultura y Deporte (2013).

13. El término utilizado por el Ministerio es el de inversión.

14. Institución de educación superior técnica, no universitaria.

15. http://www.expansion.com/emprendedoresempleo/desarrollocarrera/2015/06/19/55843d2aca474122548b45a3 .html

16. http://manecolombia.blogspot.com/2013/02/rechazamos-lainjerencia-del-banco.html 
17. Crédito ACCES: Acceso con Calidad a la Educación Superior

18. Sistema para la Prevención de la Deserción en las Instituciones de Educación Superior.

\section{Recibido: 12-noviembre-2015}

Aceptado: 11-febrero-2016

Todos los derechos reservados. Universidad de Costa Rica. Esta revista se encuentra licenciada con Creative Commons ReconocimientoNoComercial-SinObraDerivada 3.0 Costa Rica. (CC BY-NC-SA 3.0 CR)

Correo electrónico: humanidades@ucr.ac.cr Sitio web: http://revistas.ucr.ac.cr/index.php/humanidades 